\title{
A Mid- to Long-Term Results of The Avascular Fibular Grafting in ONFH With 6 to 8-Year Follow-Up
}

Peng Yuan

Nanjing University of Traditional Chinese Medicine: Nanjing University of Chinese Medicine

\section{Bin Du (D839512583@qq.com)}

Nanjing University of Traditional Chinese Medicine: Nanjing University of Chinese Medicine https://orcid.org/0000-0002-5943-0860

\section{Xin Liu}

Affiliated Hospital of Nanjing University of Chinese Medicine: Jiangsu Province Academy of Traditional Chinese Medicine

\section{Guang-Quan Sun}

Affiliated Hospital of Nanjing University of Chinese Medicine: Jiangsu Province Academy of Traditional Chinese Medicine

\section{Xuan-Ye Lin}

Nanjing University of Traditional Chinese Medicine: Nanjing University of Chinese Medicine

\section{Xu Wang}

Nanjing University of Traditional Chinese Medicine: Nanjing University of Chinese Medicine

\section{Research article}

Keywords: ARCO, ONFH, THA

Posted Date: September 30th, 2020

DOI: https://doi.org/10.21203/rs.3.rs-81466/v1

License: (c) (i) This work is licensed under a Creative Commons Attribution 4.0 International License.

Read Full License 


\title{
1 A Mid- to long-term Results of The Avascular Fibular Grafting in
}

2 ONFH with 6 to 8- year Follow-up

\author{
Peng Yuan, Bin Du, Xin Liu, Guang-Quan Sun, Xuan-Ye Lin, Xu Wang
}

4

\section{Abstract}

Background: Free vascularized fibular grafting was reported a favorable method to prevent the collapse of the femoral head. Nevertheless, this operation is traumatic and expensive. This study analyzed the long-term outcomes of avascular fibular grafting for osteonecrosis of the femoral head(ONFH) with 6 to 8 year follow-up.

Methods: From March 2009 to March 2012, 34 patients(48 hips) were diagnosed the ONFH with Association Research Circulation Osseous(ARCO) stage of II to IIIb and operated with avascular fibular grafting in Jiangsu Province Hospital of traditional Chinese Medicine. We retrospectively reviewed the clinic effects of these patients and assessed the difference effects with diverse stages(ARCO) and types(China-Japan Friendship Hospital classification, CJFH) annually. The hip survival rate, Harris hip score, activity level and imaging stability were calculated. The average follow-up time was 6.7 years.

Results: At the last follow-up, 34 of 48 hips survived totally. Depending on the ARCO stage, The overall clinical success rate for hip preserving were $76.0 \%(19 / 25)$ in II stage , $85.7 \%(12 / 14)$ in IIIA stage and 33.3\%(3/9) in IIIB stage. Referring to the CJFH classification system, the hip survival rate were $100 \%(2 / 2)$ in M type,90.9\%(10/11) in C type, $77.8 \%(14 / 18)$ in L1 type,57.1\%(8/14) in L2 type and $0 \%(0 / 3)$ in L3 type. The mean VAS score, HHS, and WOMAC were significantly improved at the final follow-up compared with preoperative values $(\mathrm{p}<0.001)$. 
Conclusion: The avascular fibular grafting operation can increase the hip function and improve patients' lives quality. It possesses vast clinical as well as practical significance, because the long-term efficacy can satisfy fundamental life requirements, especially for those early-stage and small-scale patients who suffer ONFH to avoid or put off the time of total hip arthroplasty(THA) surgery.

\section{Introduction}

Osteonecrosis of the femoral head (ONFH) is often with a progressive course, which primarily affects young adults and finally necessitates total hip arthroplasty (THA)[1].Various treatment protocols for ONFH have been attempted with diverse indications and prognoses, such as medullary core decompression, fibular support surgery, bone flap grafting, osteotomy and some medicines for instance enoxaparin[2]. Nevertheless, it is no doubt that the preservation of the femoral head for several years or more remains the main goal of treatment for young patients.

In recent years, the free vascularized fibular grafting (FVFG) has been shown as a valuable operation for dealing with ONFH by providing revascularization, mechanical support and dynamic cells to the femoral head[3].The traditional technique of this surgical management of ONFH, in which the vascular pedicle is dissected prior to osteotomy in the harvest of the vascularized fibular graft, the process may have some disadvantages including increase of surgery time, microvascular anastomosis, and risk of the proximal femoral fracture[4].

Avascular fibular grafting(AVFG) for osteonecrosis of the femoral head was used widely in the past two decades[5].The advances of this operation included smaller 
trauma(without vascular anastomosis and usually use the allograft), simple technology, less complications and lower expense[6]. Researchers had reported the outcomes of a vascularized fibular grafting but the outcomes were various[7-10].Plenty of literature researches reported that with choosing strictly indications the AVFG might have ideal clinic outcomes as well as FVFG[11].Cheng-Ta Wu et al. conducted research regarding a analysis of the necrosis of femoral head with ARCO stage II to stage IIIB that had underwent Non-vascularized bone grafting operation for the treatment. At the final follow-up, 29 hips in 24 patients showed $88.5 \%$ of five year survival rate and $76.9 \%$ of 12 -year survival rate using conversion to THA as the end point[12]. Another scholar compared the autograft implantation to the bone grafting through fenestration over femoral head and neck with a 8months' to 40months' follow-up. Stage IIB-IIIA according to the classification system of ARCO were selected in this study. At the last follow up, the Harris hip aggregate score, pain score and range of motion score of patients under autograft implanting were all significantly superior to the bone grafting group[13]. Just like the study above, our experiences of clinical treatment showed that with thoroughly clearing the sequestrums and tightly impacted bone grafting, the avascular fibular grafting operation can provide potent supporting for the femoral head to avoid the femoral head collapse. Our study aimed to assess the medium to long term clinical and radiological outcomes for this sort of surgery in patients of ARCO stage II to IIIB and explore the optimum indications of this operation.

\section{Materials and Methods}

We retrospectively reviewed our database of Jiangsu Province Hospital of Traditional 
Chinese Medicine during March 2009 to March 2012 and identified 34 patients with 48 AVFG operations , whose stage were at ARCO stage II to IIIb of ONFH. This study included age younger than 55 years old. All patients inserted with using the same standard instruments and all operations were performed by a single, experienced surgeon. The study objects consisted of 29 males with 40 hips and 5 females with 8 hips. Pathogenic factors included using glucocorticoid continuously, long-term alcohol consumption, trauma, hypercoagulable state(such as anticardiolipin antibodies syndrome) and idiopathic cases. Functional disorder of hip and hip pain occurred in all patients. The diagnosis of ONFH was based on symptoms, physical signs, previous relative medical history and relevant risk factors. In addition, anteroposterior(AP) and frog-leg lateral(FL) views of bilateral hip, Computed Tomography (CT), and Magnetic Resonance Image (MRI) were generally used in each patient to confirm the diagnosis and to refine the type. Diagnostic criteria are "Guidelines for the Clinical Diagnosis and Treatment of adult femur head necrosis (2019 edition)" [14]. The patients' background and details are listed in Table 1.

Surgery was all performed under general anesthetic. Each patient were positioned supine with a bolster under the ipsilateral buttock. When sterilization done, a 7 to 8 $\mathrm{cm}$ length of incision along the Watson-Jones approach upper to the greater trochanter was performed. Once the greater trochanter was exposed, we performed the core decompression of the femoral head. Two cm down-ward to the tip of the greater trochanter, we inserted a $2.5 \mathrm{~mm}$ Kirschner pin toward anterior-lateral necrotic area of 
the femoral head till $0.5 \mathrm{~cm}$ underneath the subchondral bone. When the Kirschner pin positioned correctly under the C-arm X-ray machine, we detected the length of Kirschner pin inserted in the femoral neck and head, a matched self-designed hollow reamer was used to broach the tunnel along the pin till $0.5 \mathrm{~cm}$ below the subchondral bone. We applied different diameter of "T" shape hand driller to broach the tunnel to 10 to $12 \mathrm{~mm}$ which was determined by the allograft non-vascularized fibular bone maximal breadth. The necrotic bone must be removed along the tunnel with a curet, especially in the lateral loading area. Subsequently, we performed tightly impaction bone grafting to the necrotic area with allogeneic bone granule which was loaded into a self-designed plugger. The crucial techniques of tightly impaction bone grafting were layer-by-layer and $360^{\circ}$ impaction with differ sizes straight and curved head impaction rod. The standard criterion of complete impaction bone grafting was that the density of the necrotic bone was equally increased and restoration of the femoral head normal shape under C-arm. Before allogeneic fibular implantation, we drilled bi-cortical crossed holes at $0.5 \mathrm{~mm}$ interval vertical to the axial of the allograft fibular with $2 \mathrm{~mm}$ Kirschner pin which was considered to be beneficial to the new formed repairmen bone creeping substitution. (Figure 1)And the tip of the allograft fibular was blunt and $40^{\circ}$ slope processed with bone file which can decrease the mechanical stress through the increasing bony contact. The predisposed allograft fibular was inserted tightly into the well-prepared tunnel with the impaction rod till $0.5 \mathrm{~cm}$ below the subchondral bone through fluoroscopy and the protrude end was trimmed with bone rongeur. We closed the incision after complete sterile saline solution wash which 
112

113

prevent the heterotopic ossification in the surgical site and keep the drainage tube for 2 days. Weight-bearing was strictly prohibited after surgery and the quadriceps and iliopsoas muscles were trained in bed. X-ray was taken after 3 months for reexamination. If the internal structure of femoral head was stable, partial load can be carried out with a walking aid. Six months after surgery, the full weight-bearing was permitted.

\section{Statistical analysis}

The pre- and post-treatment data within the same group were compared statistically using the Kruskal-Wallis test and Kaplan-Meier method. The data is represented by medians and confidence intervals. All data was processed through SPSS16.0 and p value less than 0.01 was considered as statistical significance.

\section{Results}

34 hips performed well in all 48 hips followed up, which arthroplasty surgery was not required in the short term. Most of the femoral heads with successful hip preservation showed stable repairing in the head. About half (14/34) of the imaging showed slight collapse $(\leqslant 1 \mathrm{~mm})$, but with no clinic symptoms. These femoral heads were characterized by slight collapse, but the subchondral bone is well bonded to the repair area and is therefore structurally stable. All the hip joints with good efficacy had significant high density repair bands in the compression zone of bone graft. The restoration band edges were well integrated with the surrounding bone trabeculae, and there were no obvious bright lines around the restoration zone (Figure 2). The 
proportion of imaging stable repair was well correlated with preoperative CJFH classification and was also associated with ARCO stage and necrotic area[15-16].

During an average of 6.7 years follow-up, Kaplan-Meier survival curve of the patients included in this study was consistently associated with preoperative ARCO stage (Figure 3).At 2-years follow-up, patients of ARCO stage were without fail completely. But the survival rate of hips in this study were not exactly presenting positive correlation with ACRO stage. At the last follow-up, depending on the ARCO stage, the hip survival rate were $76.6 \%$ (19/25) in II stage,85.7\%(12/14) in IIIA stage and $33.3 \%(3 / 9)$ in IIIB stage (Table 2) .Compared to ARCO stage, Kaplan-Meier survival curve of hip joint during follow-up in this study had a better correlation with preoperative CJFH classification system (Figure 4). Referring to the CJFH classification system, the hip survival rate were $100 \%(2 / 2)$ in M type, $90.9 \%(10 / 11)$ in C type, $77.8 \%(14 / 18)$ in $\mathrm{L} 1$ type, $57.1 \%(8 / 14)$ in $\mathrm{L} 2$ type and $0 \%(0 / 3)$ in $\mathrm{L} 3$ type at the last follow-up (Table 2) .

After avascular fibular grafting, the majority of patients reported considerable relief of hip pain at night and better hip motion in daily activity and work. we found that the VAS score, HHS, and WOMAC of the patients were significantly improved compared to pre-operation (Table 3 ).The patients were basically painless and had good hip function in all directions in short-term follow-up. In this study, pain was the main factor leading to failure of hip preservation, followed by decreased painless activity 
ability, especially the painless walking distance less than 200 meters, which was disastrous for relatively young patients. During multiple postoperative follow-up visits, we found that the average Harris score of patients within 4 years decreased with the extension of follow-up time, and all failed hip preservation (14/48) underwent hip replacement within 4 years after surgery. However, no more hip preservation failure occurred from 4-year follow-up to the last follow-up in our study, and during this period the Harris score was basically stable (Figure 5). Since some of the patients in this study had bilateral disease, any lesions on either side may affect pain and motor function score, causing Harris score to be interfered, so we conducted the intraoperative hip joint mobility separately, and evaluated the level of postoperative joint activity by percentage method to reflect the degree of joint matching. The results showed that the level of postoperative hip joint activity was significantly improved (Figure 5).

\section{Discussions}

Postponing or avoiding hip replacement has positive significance in younger patients with femoral head necrosis. At present, it is believed that the femoral head necrosis is difficult to complete the self-repair by two main factors: blood transport disorder and mechanical collapse[17].Based on this pathophysiological understanding, it is obvious that the main objective of hip preservation surgery should be to restore blood flow in the head and maintain mechanical stability.

In hip preservation operation methods, free vascularized fibular graft is considered to 
provide good arterial blood supply in the head through anastomosis of vessels while providing support. The clinical efficacy of this surgery had been reported in many literatures, but its efficacy varies greatly among different reports. The reason for this difference may be that the surgical method has higher requirements on the surgical technique and the surgeons' different understanding of indications. At present, it is relatively certain that the early and middle stage femoral head necrosis before collapse may have higher intermediate and long term success rate[18].William C et al. used vascularized fibular graft in a clinical study on long-term efficacy of femoral head necrosis in the early and middle period before collapse. Among them, 49 of 65 hips (75\%) had surviving for at least 10 years. At last follow-up, 39 of 65 hips (60\%) had surviving FVFG, with a mean graft survival time of 15 years(range, 10.5-26.1 years). 26 of the 65 hips with FVFG (40\%) underwent conversion to THA at a mean of 8 years postoperatively[19].Another large sample clinical study using a vascularized fibular graft for early to mid-late femoral head necrosis, a total of 407 patients(518 hips) were included. The follow-up ranged from 3 to 10 years with an average of 5.0 years. In comparison with the preoperative data, the radiographic evaluation showed no changes in 255 patients (331 hips, 57.3\%), improvement in 116 patients (195 hips, $33.7 \%$ ), and progression in 36 patients (52 hips, 9.0\%). Among the patients with progression status, 20 patients (23 hips) had total hip arthroplasty[20].In this study, we used the minimally invasive avascular fibular grafting treating the ARCO stage II to IIIB patients, for an average follow-up of 6.7 years, the total survival rate reached $70 \%(34 / 48) .6$ of the 14 collapse cases were IIIB stage, to eliminate which the success 
rate reached $79.5 \%(31 / 39)$. According to the CJFH classification, in addition to the full head necrosis of the L3 type all 3 cases confirmed failure (which 3 cases were all ARCO IIIB stage), the overall hip preservation success rate of other types reached $75.5 \%(34 / 45)$. These data above were not inferior to those with vascularized fibula grafts. Therefore, for the advanced femoral head necrosis with incomplete-head type, the avascular allograft fibular transplantation may achieve the same good long-term hip preservation effect as the vascular fibular transplantation, and has the advantages of less trauma, shorter operative time and fewer complications.

In this study, we found that in general, the long-term success rate of hip preservation by using a vascularized fibular was relatively ideal. The main factor affecting clinical efficacy were choosing suitable indications. According to this study, based on the ARCO stage, the long-term success rate of hip preservation were IIIA $>$ II $>$ IIIB. The cause of stage IIIA had a better curative effect than that of II may due to dead bone cleaning scope of this surgery was relatively small and the effectively support area of fibula was limited. In addition, the granule bone grafting may has an advantage for small-scale bone repair. By radiographic follow-up, we found that although the IIIa stage had been a slight collapse under the cartilage, but by clearing the dead bone and pressing the bone graft tightly, the collapse of the cartilage surface can be restored to a certain extent, and long-term stability can be achieved in imaging. Therefore, this surgery can still obtain ideal curative effect for patients which had mild collapse but with relatively small necrosis area. The success rate of hip preservation based on 
CJFH classification was $\mathrm{M}>\mathrm{C}>\mathrm{L} 1>\mathrm{L} 2>\mathrm{L} 3$. Necrosis of the lateral column and large area of necrosis were main factors associated with poor prognosis. Three patients of type L3 with extensive femoral head necrosis all had hip replacement within 2 years. We do not recommend this surgery for these patients.

\section{Conclusions}

The avascular fibular grafting operation can increase the hip function and improve patients' lives quality. For proper indications and understanding key points, the operation has ideal long-term clinical effect. At the same time, compared to vascularized fibular graft, this operation is relatively simple and the complications are seldom.

\section{Abbreviations}

ONFH: Osteonecrosis of femoral head; ARCO: Association Research Circulation Osseous; CJFH: China-Japan Friendship Hospital classification; VAS: Visual analog scale; WOMAC: Western Ontario McMaster Osteoarthritis index; HHS: Harris hip score; THA: total hip arthroplasty; FVFG: free vascularized fibular grafting; AVFG: Avascular fibular grafting

\section{Acknowledgements}

We want to express our sincere appreciation for all the patients that joined this study. 


\section{Authors' contributions}

245 Peng Yuan was responsible for data analysis and manuscript writing. Xin Liu

246 contributed to data collection and language editing. Guang-Quan Sun, Xuan-Ye Lin,

247 and Xu Wang contributed to the data collection. Bin Du was responsible for the study

248 design and correspondence. All authors read and approved the final manuscript.

249

$250 \quad$ Funding

251 The author(s) received no financial support for the research, authorship and/or

252 publication of this article.

253

254 Availability of data and materials

255 The datasets used and/or analyzed during the current study are available

256 from the corresponding author on reasonable request.

\section{Ethics approval and consent to participate}

259 The study was approved by the Clinical Trials and Biomedical Ethics Committee of

260 Affiliated hospital of Nanjing University of Chinese Medicine, and written informed

261 consent was obtained from all participants. All experiments were performed in

262 accordance with relevant guidelines and regulations.

\section{Consent for publication}

265 All authors have seen the manuscript and approved it to submit to your journal. 


\section{Declaration of conflicting interests}

268 The author(s) declared no potential conflicts of interest with respect to the research,

269 authorship, and/or publication of this article.

270

$271 \quad$ Reference

272 1. Zalavras CG, Lieberman JR. Osteonecrosis of the femoral head: evaluation and

273 treatment. J Am Acad Orthop Surg. 2014;22(7):455-464.

274 2.Tripathy SK, Goyal T, Sen RK. Management of femoral head osteonecrosis: Current 275 concepts. Indian J Orthop.2015 Jan-Feb;49(1):28-45.

276 3. Ryan SP, Wooster B, Jiranek W, Wellman S, Bolognesi M, Seyler T. Outcomes of

277 Conversion Total Hip Arthroplasty From Free Vascularized Fibular Grafting. J

278 Arthroplasty. 2019;34(1):88-92.

279 4.Meloni MC, Hoedemaeker WR, Fornasier V. Failed vascularized fibular graft in

280 treatment of osteonecrosis of the femoral head. A histopathological analysis. Joints.

2812016 Jun 13;4(1):24-30.

282 5. Lakshminarayana S, Dhammi IK, Jain AK, Bhayana H, Kumar S, Anshuman R.

283 Outcomes of Core Decompression with or without Nonvascularized Fibular Grafting

284 in Avascular Necrosis of Femoral Head: Short Term Followup study. Indian J Orthop.

285 2019;53(3):420-425.

286 6. PN Soucacos, AE Beris, K Malizos, A Koropilias, H Zalavras, Z

Dailiana.Treatment of avascular necrosis of the femoral head with vascularized fibular 
transplant. Clin Orthop Relat Res. 2001 May;(386):120-30.

7.Moya-Angeler J, Gianakos AL, Villa JC, Ni A, Lane JM.Current concepts on osteonecrosis of the femoral head. World J Orthop. 2015 Sep

18;6(8):590-601.8.Kuroda Y, Matsuda S, Akiyama H. Joint-preserving regenerative therapy for patients with early-stage osteonecrosis of the femoral head. Inflamm Regen. 2016 Apr 25;36:4.

9. Gou WL, Lu Q, Wang X, Wang Y, Peng J, Lu SB. Key pathway to prevent the collapse of femoral head in osteonecrosis. Eur Rev Med Pharmacol Sci. 2015 Aug;19(15):2766-74.

10. Mao Q, Wang W, Xu T, Zhang S, Xiao L, Chen D, Jin H, Tong P. Combination treatment of biomechanical support and targeted intra-arterial infusion of peripheral blood stem cells mobilized by granulocyte colony stimulating factor for the osteonecrosis of the femoral head: A randomized controlled clinical trial. J Bone Miner Res. 2015 Apr;30(4):647-56.

11. Changjun C, Donghai L, Xin Z, Liyile C, Qiuru W, Pengde K. Mid- to long-term results of modified non-vascularized allogeneic fibula grafting combined with core decompression and bone grafting for early femoral head necrosis. J Orthop Surg Res. 2020;15(1):116. Published 2020 Mar 24.

12. Wu CT, Yen SH, Lin PC, Wang JW. Long-term outcomes of Phemister bone grafting for patients with non-traumatic osteonecrosis of the femoral head. International Orthopaedics. 2019 Mar;43(3):579-587.

13. Keizer SB, Kock NB, Dijkstra PD, Taminiau AH, Nelissen RG.Treatment of 
avascular necrosis of the hip by a non-vascularised cortical graft. J Bone Joint Surg Br . 2006 Apr;88(4):460-6.

14. Zhao D, Zhang F, Wang B, Liu B, Li L, Kim SY, Goodman SB, Hernigou P, Cui Q, Lineaweaver WC, Xu J, Drescher WR, Qin L. Guidelines for clinical diagnosis and treatment of osteonecrosis of the femoral head in adults (2019 version). J Orthop Translat. 2020;21:100-110. Published 2020 Jan 6.

15. Yoon BH, Mont MA, Koo KH, Chen CH, Cheng EY, Cui Q, Drescher W, Gangji V, Goodman SB, Ha YC, Hernigou P, Hungerford MW, Iorio R, Jo WL, Jones LC, Khanduja V, Kim HKW, Kim SY, Kim TY, Lee HY, Lee MS, Lee YK, Lee YJ, Nakamura J, Parvizi J, Sakai T, Sugano N, Takao M, Yamamoto T, Zhao DW.The 2019 Revised Version of Association Research Circulation Osseous Staging System of Osteonecrosis of the Femoral Head. J Arthroplasty. 2020;35(4):933-940. 16. Xia T, Wei W, Zhang C, Ji W, Shen J. Zhongguo Xiu Fu Chong Jian Wai Ke Za Zhi. 2020;34(1):10-15.

17. Calori GM, Mazza E, Colombo A, Mazzola S, Colombo M.Core decompression and biotechnologies in the treatment of avascular necrosis of the femoral head.EFORT Open Rev.2017 Mar 13;2(2):41-50.

18. Fang T, Zhang EW, Sailes FC, McGuire RA, Lineaweaver WC, Zhang F.Vascularized fibular grafts in patients with avascular necrosis of femoral head: A systematic review and meta-analysis.Arch Orthop Trauma Surg. 2013 Jan;133(1):1-10.

19. William C Eward 1, Craig A Rineer, James R Urbaniak, Marc J Richard, David S 
Ruch.The Vascularized Fibular Graft in Precollapse Osteonecrosis-Is Long-term Hip Preservation Possible?Clin Orthop Relat Res.2012 Oct;470(10):2819-26.

20. Gao YS, Chen SB, Jin DX, Sheng JG, Cheng XG, Zhang CQ.Modified Surgical Techniques of Free Vascularized Fibular Grafting for Treatment of the Osteonecrosis of Femoral Head: Results from a series of 407 cases.Microsurgery.2013

Nov;33(8):646-51.

Table 1: Demographic characteristic of the patients

Table 2: Difference of survivorship among the groups

Table 3: Baseline and outcomes of the last follow-up

Figure 1:The density of compression implant should exceed the surrounding cancellous bone (arrow a). The hole was drilled with Kirschner's needle (arrow b), Fill the outside of the passage to make it jam (arrow c).

Figure 2:The patient's preoperative AP/FL film showed obvious sclerosing zone (A/B).6 years after surgery, no collapse was found, and a high-density restoration zone was found (C/D).CT shows that the implant has formed good integration with the surrounding bone $(\mathrm{E})$.

Figure 3: Kaplan-Meier survival curve according to ARCO stage.

Figure 4: Kaplan-Meier survival curve according to CJFH classification.

Figure 5: Relationship between Harris score and follow-up time(green dotted line).Relationship between Harris activity level and follow-up time(yellow dotted line). 


\section{Figures}

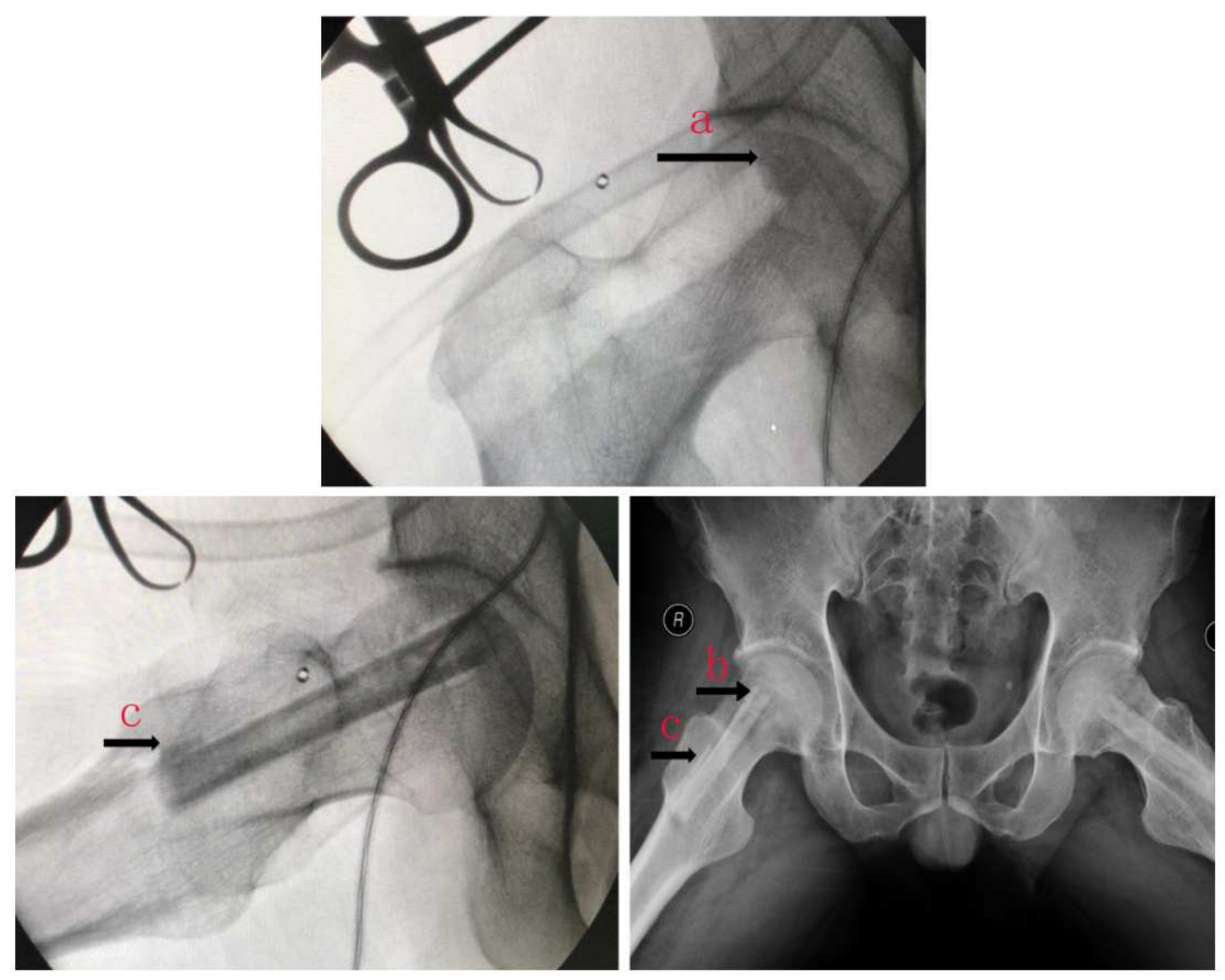

Figure 1

The density of compression implant should exceed the surrounding cancellous bone (arrow a). The hole was drilled with Kirschner's needle囚arrow b) $₫$ Fill the outside of the passage to make it jam (arrow c). 

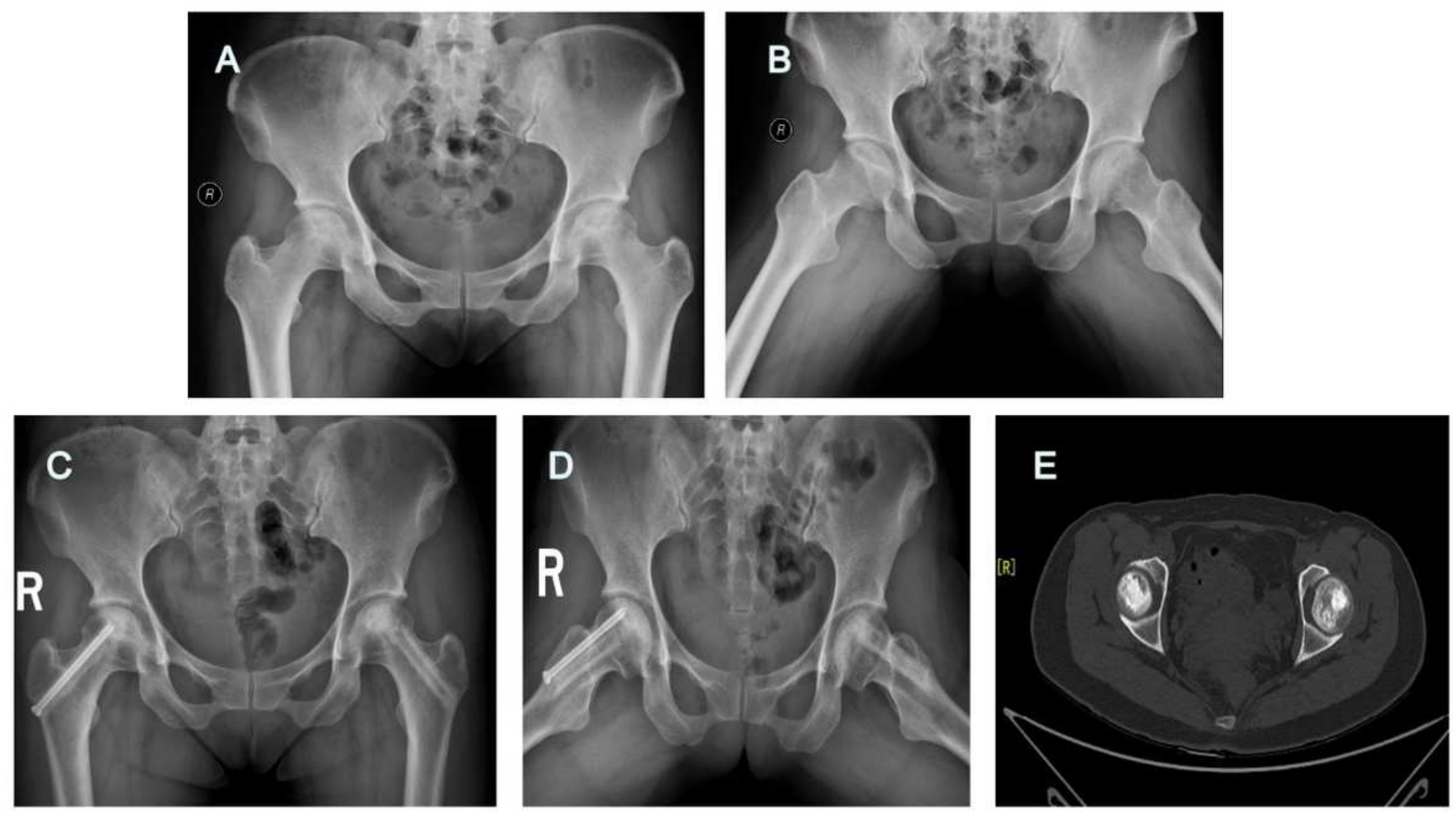

Figure 2

The patient's preoperative AP/FL film showed obvious sclerosing zone (A/B). 6 years after surgery, no collapse was found, and a high-density restoration zone was found (C/D).CT shows that the implant has formed good integration with the surrounding bone $(E)$. 


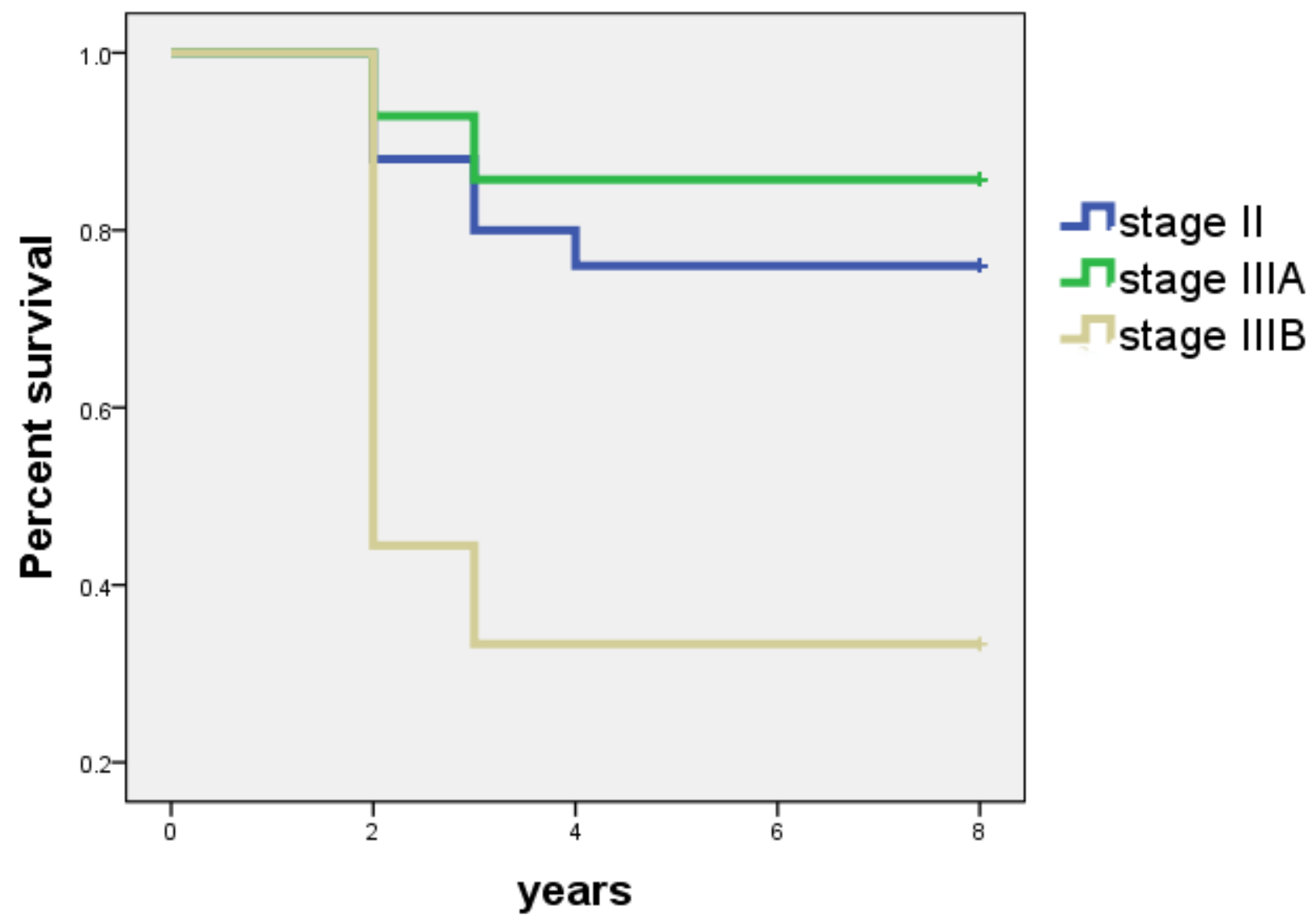

Figure 3

Kaplan-Meier survival curve according to ARCO stage. 


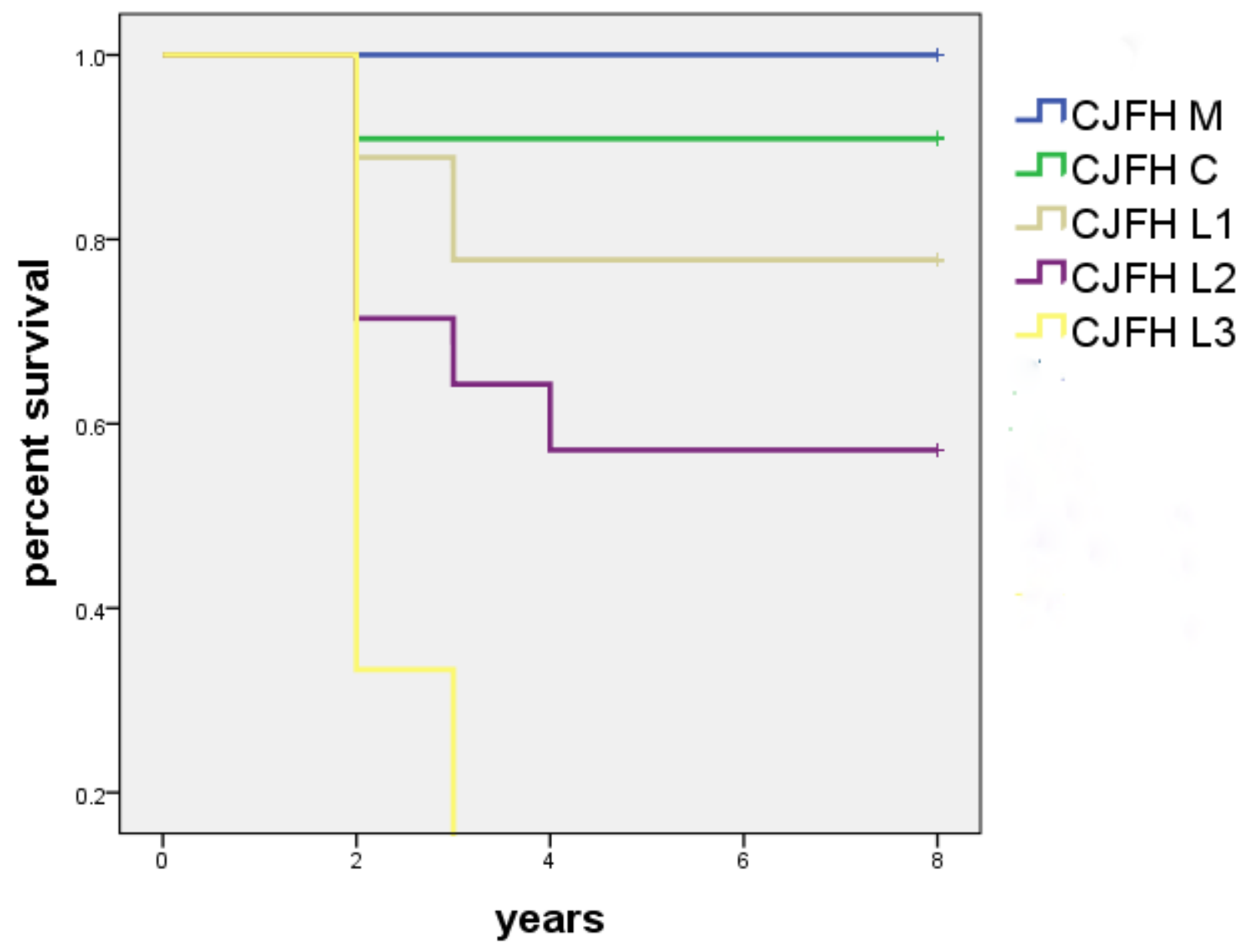

Figure 4

Kaplan-Meier survival curve according to CJFH classification. 


\section{Harris Score}

\section{Activity level(Score/5 × 100\%)}

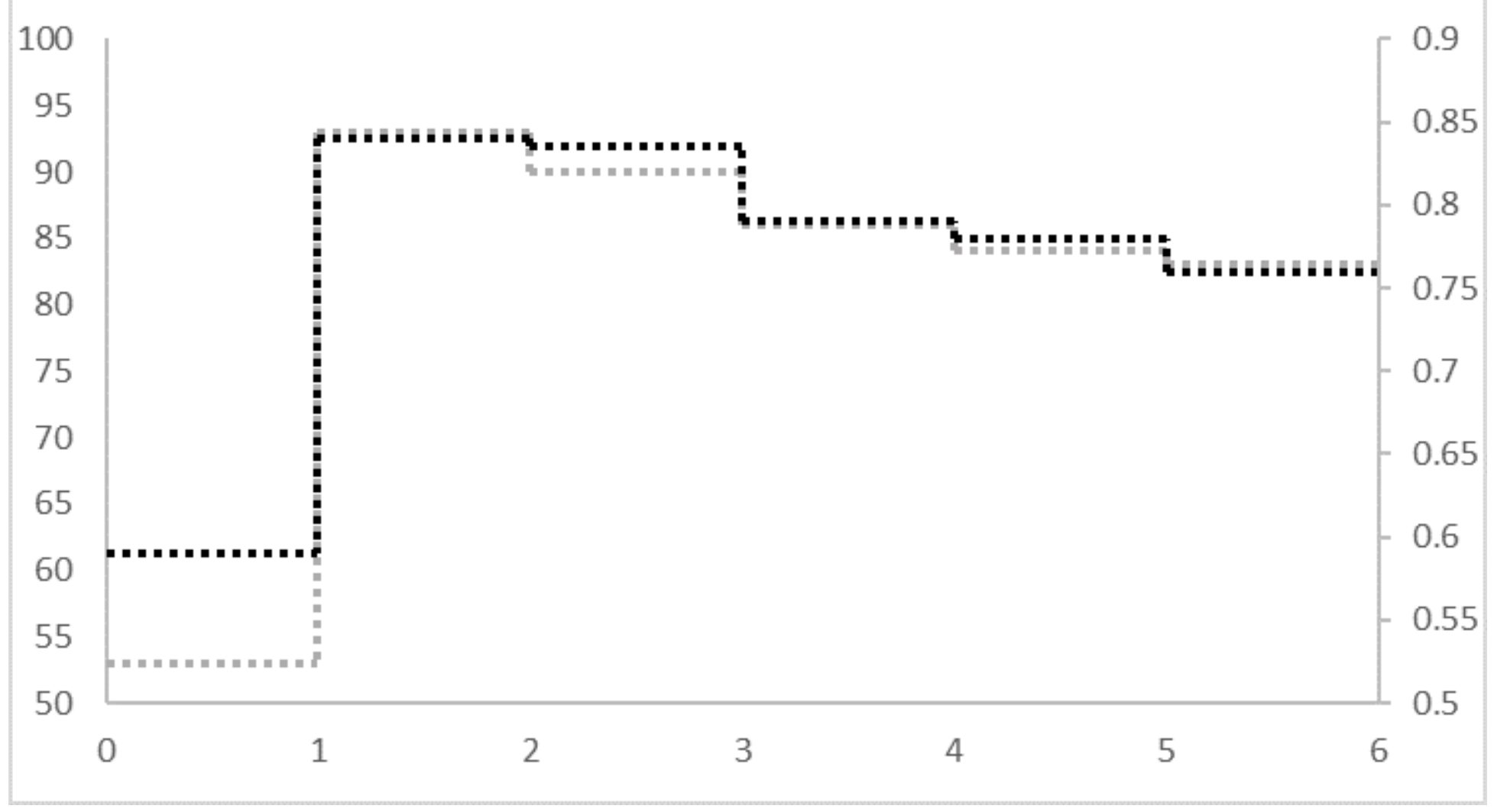

Figure 5

Relationship between Harris score and follow-up time(green dotted line). Relationship between Harris activity level and follow-up time(yellow dotted line).

\section{Supplementary Files}

This is a list of supplementary files associated with this preprint. Click to download.

- table1.png

- table2.png

- table3.png 